\title{
MRC B1221-423: Forming an AGN in a merger
}

\author{
Helen Johnston ${ }^{1}$, Richard Hunstead ${ }^{1}$, Elaine Sadler ${ }^{1}$, Garret Cotter ${ }^{2}$ \\ and Raffaella Morganti ${ }^{3}$ \\ ${ }^{1}$ School of Physics, University of Sydney, NSW 2006, Australia \\ ${ }^{2}$ Department of Astrophysics, University of Oxford, Oxford OX1 3RH, UK \\ ${ }^{3}$ NFRA, Postbus 2, NL-7990 AA, Netherlands
}

\begin{abstract}
MRC B1221-423 is a powerful, nearby $(z=0.17)$ compact radio source, which lies within the envelope of a galaxy which is undergoing a tidal interaction with a companion. Broad-band optical/NIR colours show evidence of several distinct episodes of star formation. The evidence points to this being a radio source "caught in the act" of its first ignition.
\end{abstract}

There is growing evidence for a link between starbursts and the onset of radio-loud AGN activity, possibly triggered by galaxy mergers. This merger activity is expected to lead to large increases in the amount of material being fed to the central black hole, thereby triggering the strong radio emission.

Compact steep-spectrum (CSS) sources are ideal candidates for testing the connection. These are powerful radio sources which are entirely contained within their host galaxy, and the consensus is that they are young (O'Dea 1998). CSS sources are nearly always found in interacting systems, suggesting there may be a close connection between the formation of the AGN and the merger.

MRC B1221-423 is one of the nearest CSS sources, located in a galaxy at redshift $z=0.1706$. It has a steep spectrum $\left(\alpha=-0.85, S_{\nu} \propto \nu^{\alpha}\right)$, and double lobes separated by only 1 .' $5(4.3 \mathrm{kpc})$. The age of the source, estimated from the energy density of the jets, is only $10^{5} \mathrm{yr}$ (Safouris et al. 2003).

We have detected strong HI absorption against the radio source at an infall velocity of about $200 \mathrm{~km} \mathrm{~s}^{-1}$ (Fig. 1). This continues a trend in which all galaxies with recent star formation show HI absorption, in contrast to "normal" radio galaxies, which only rarely show HI absorption (Morganti et al. 2004).

Five-colour BVRIK images of B1221-423 show a probable companion and tidal tail, along with faint shells, which are clear signs of an ongoing interaction (Johnston et al. 2003). The colour-magnitude diagram of individual pixels of B1221-423 shows strong features, which map to distinct spatial regions in the images (Fig. 2). The most obvious interpretation is that the three regions represent different stellar populations, products of separate episodes of star formation.

We can also fit stellar population models to the pixel spectra energy distributions. An input population is chosen and fixed at the known redshift of the galaxy $(z=0.1706)$. A grid of model fluxes, with varying age and reddening, is compared to the observed BVRIK fluxes for each pixel, and the best fit age and reddening is recorded. The ages fall into three distinct groups, with ages of around $13 \mathrm{Gyr}, 500 \mathrm{Myr}$ and $10 \mathrm{Myr}$. Plotting the location of pixels of different ages, the ages clearly correlate with the features of the interacting system. The old population is found throughout the galaxy, the intermediate age population is found in the companion and tidal tail, while the youngest pixels are in the nucleus, the blue knots, and the companion. 

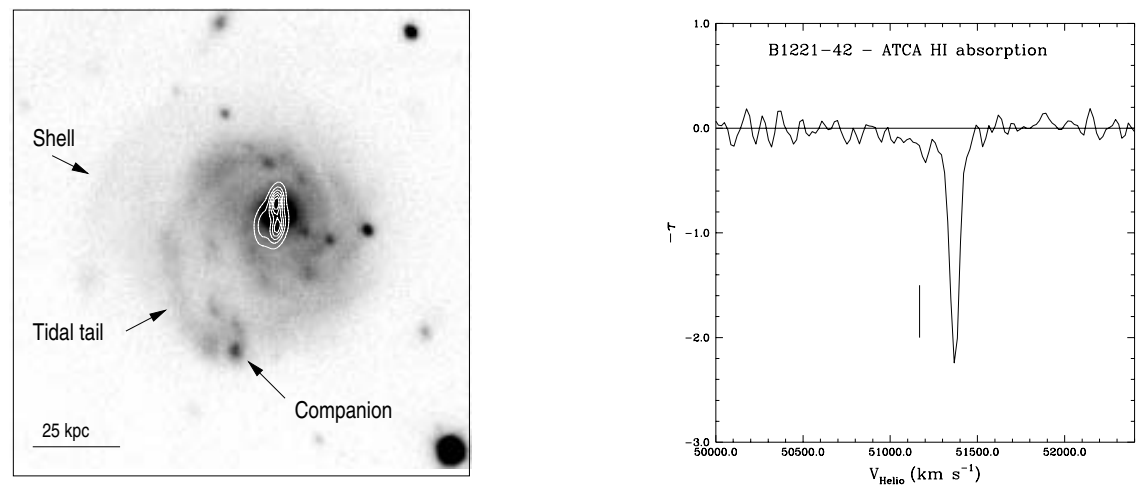

Figure 1. (left) $R$-band image, overlain with radio contours to show the scale of the radio source. (right) HI absorption against the radio source, showing large amounts of infalling material.
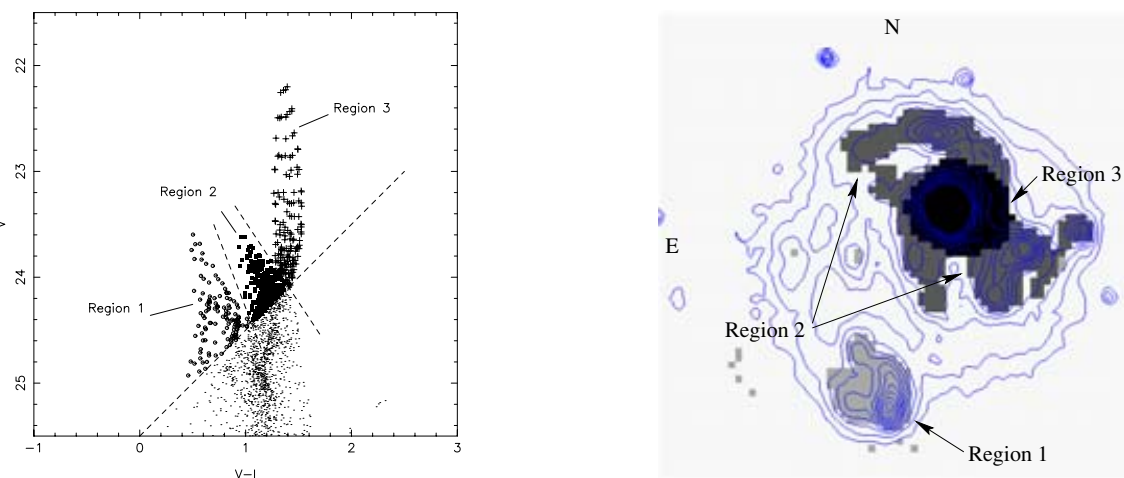

Figure 2. "Plumes" of pixels in the $V$ vs. $V-I$ colour-magnitude diagram (left) correspond to distinct regions of the galaxy (right). Contours show $B$-band intensity.

Thus we have evidence for three distinct episodes of star formation in B1221-423. These episodes are clearly correlated with position in the system, and hence are most likely triggered by tidal interactions with the companion galaxy. The evidence points to the AGN in the centre of B1221-423 having been "caught in the act" of turning on. However, none of the components we have identified is as young as the radio source, implying that the time delay between the interaction and the triggering of the AGN is at least $3 \times 10^{8}$ years.

A possible scenario to explain the three component populations is as follows: The tidal interaction began 300 Myr ago, which triggered star formation in both the host galaxy and the companion, forming the intermediate-age population. Gas is driven down into the nucleus on timescales of $10^{8} \mathrm{y}$, where it both cools to form stars, which we see as the 10 Myr-old population, and triggers activity in the central supermassive black hole.

\section{References}

Johnston, H. M., Hunstead, R. W., Cotter, G., \& Sadler, E. M. 2003, MNRAS, submitted Morganti, R., Tadhunter, C. N., Emonts, B., Holt, J., \& Oosterloo, T. 2004, in IAU. Symposium 217, (eds. P.-A. Duc, J. Braine and E. Brinks), in press

O'Dea, C. P. 1998, PASP, 110, 493

Safouris, V., Hunstead, R. W., \& Prouton, O. R. 2003, PASA, 20, 1 